\title{
Concurrent administration of oxytocin with Foley catheter versus Misoprostol for induction of labor: A systematic review and meta-analysis
}

Mohamed A. Abdel Aziz

Benha University Faculty of Medicine

Shayeste Jahanfar

Central Michigan University College of Medicine

Parvin Abedi ( $\sim$ parvinabedi@ymail.com )

Ahvaz Jondishapour University of Medical Sciences https://orcid.org/0000-0002-6980-0693

Shahla Faal

Islamic Azad University Marand Branch

Research

Keywords: Foley catheter, Oxytocin, Misoprostol, labor induction

Posted Date: October 14th, 2020

DOI: https://doi.org/10.21203/rs.2.20722/v2

License: @ (i) This work is licensed under a Creative Commons Attribution 4.0 International License.

Read Full License 


\section{Abstract}

Background: Around one- third of pregnancies require labor induction due to several reasons such as post-term pregnancy, stillbirth, and medical complications. This systematic review aimed to evaluate the effect of concurrent administration of Foley catheter plus oxytocin versus Misoprostol on labor induction.

Methods: The search was conducted in April 2019 and updated in September 2020. The following databases were searched: PubMed, SCOPUS, Cochrane Central Register of controlled trials, and Web of Science. Primary outcome included cesarean section rate. Secondary outcomes were the mean duration of induction to delivery and delivery in less than 12 hours from induction. Data were analyzed using RevMan. For binary outcomes, the odds ratio with $95 \%$ confidence intervals $(\mathrm{Cl})$, and for continuous outcomes, the mean difference (MD) with $95 \% \mathrm{Cl}$ was measured.

Results: Four studies were included in this review. The cesarean section rate in Foley plus oxytocin was not significantly different from vaginal Misoprostol ( $\mathrm{OR}=0.95 ; 95 \% \mathrm{Cl}: 0.70,1.30)$ and $(\mathrm{OR}=0.92 ; 95 \%$ Cl: $0.66,1.29)$ respectively. Foley plus oxytocin decreased the mean duration of induction to delivery compared to vaginal Misoprostol in nuligravida ( $\mathrm{MD}=-6.74 ; 95 \% \mathrm{Cl}-9.20,-4.28)$, and increased delivery in less than 12 hours from induction $(\mathrm{OR}=2.08 ; 95 \% \mathrm{Cl}: 1.43,3.02)$.

Conclusion: Although the rate of cesarean and vaginal delivery was not different in two groups of Foley catheter plus oxytocin and vaginal Misoprostol, the mean time of induction to delivery reduced and delivery in less than 12 hours from induction increased in the Foley plus oxytocin.

\section{Background}

Labor induction or labor inducing defines as stimulation of uterine contraction before the onset of labor (1). Labor induction sometimes indicated in certain condition such as; post-term pregnancy, intrauterine fetal growth retardation, preeclampsia or eclampsia, premature rupture of membranes, fetal death, some cases of twin pregnancy, pregnancy-induced with medical problems such as diabetes and high blood pressure (2). Labor induction has dramatically increased in the United States (USA), from $9.6 \%$ in 1990 to $23.2 \%$ in 2011 (2). The success of labor induction is important since its failure may lead to higher rates of cesarean section with its subsequent complications (3).

Cervical ripening and labor induction can be done by non-pharmacological or pharmacological methods (4). Non-pharmacological methods include Foley balloon catheter (5), amniotomy, acupuncture, sexual intercourse (6), hypnosis (7) and breast stimulation (8), while the pharmacological methods include oxytocin, prostaglandins, nitric oxide, Misoprostol, and mifepristone $(9,10)$.

While pharmacological methods (mainly Misoprostol) are the most commonly used method, some clinicians use concurrent pharmacological and non-pharmacological methods to achieve a higher vaginal delivery rate and shorten the duration of induction. However, the introduction of non-pharmacological methods such as Foley catheters might have a detrimental effect in the form of chorioamnionitis, and 
neonatal sepsis. On the other side, pharmacological options might increase the risk of uterine hyperstimulation (11-13), and the evidence in this area is still not conclusive. Therefore, we aimed to assess the efficacy and safety of concurrent use of Foley catheter and oxytocin versus the Misoprostol during the process of induction of labor.

\section{Methods}

\section{Types of studies}

We included only randomized clinical trials (RCTs). The protocol of this study was registered in PROSPERO (Ref No: CRD42019133611). This systematic review was prepared according to Preferred Reporting Items for Systematic Reviews and Meta-Analyses (PRISMA) guidelines.

\section{Types of participants}

Women at any age with a singleton full-term pregnancy who delivered by induction of labor were included in the study.

\section{Types of interventions}

Trials included in the study were those comparing use of oxytocin in combination with Foley catheter placement for induction of labor compared to Misoprostol alone administrated by any route and by any dose. No restrictions were made regarding language, geographic area of the study, or publication date.

\section{Types of outcomes}

Primary outcomes: Primary outcomes was cesarean section rate. The CS rate was defined as the number of CS divided by the total deliveries as defined by each included study.

\section{Secondary outcomes}

Secondary outcomes were; mean duration of induction to delivery, delivery in less than 12 hours from induction, and adverse events.

\section{Adverse events}

Adverse events included hyperstimulation, chorioamnionitis, neonatal admission to neonatal intensive care unit (NICU), and neonatal sepsis.

\section{Collection and review data}

We followed the Cochrane Collaboration reviewed methods for collection and analysis of summary data $(14,15)$. 
The search was conducted on April 2019 and updated in September 2020. The following databases were searched: PubMed, SCOPUS, Cochrane Central Register of controlled trials (Issue 4 of 12, April 2019) and Web of Science. We also have searched the references of the relevant articles to detect any study that was not retrieved from our primary search. No limitations were set regarding the year of publication, language, or route and dose of the medications. The Search terms included: Misoprostol, Foley catheter, oxytocin and induction of labor (See Supplemental Material No. 1).

Search results from the databases were imported to Endnote X7 (Thompson Reuter, CA, USA) and the duplicates were removed. Two review authors (MAA and SF) independently examined all titles and abstracts according to the inclusion criteria. Any discrepancy was resolved by discussion or consideration of a third person (PA). Using a predesigned data extraction sheet, two review authors (MAA and SF) independently extracted the data. Any discrepancy was resolved by discussion. One review author (MAA) entered the data into Review Manager 5.3. A second author checked the data (SF).

When there was any data missing in included studies, we tried to contact the authors to get relevant missing data. In case the data were not provided, we applied an intention-to-treat analysis.

\section{Risk of bias assessment}

Two review authors (MAA and SF) assessed the risk of bias in each included study. Any disagreement was resolved by discussion. Risk of bias assessment was performed according to the Cochrane Collaboration reviewed methods for collection and analysis of summary data (15). For the included study, the following domains were assessed: random sequence generation, allocation concealment, blinding of participants and personnel, blinding of outcome assessment, incomplete outcome data, selective outcome reporting, and other biases (16). For each domain of the quality assessment components, it was coded as low, medium, or high risk of bias based on the author's judgment.

\section{Measures of treatment effect}

For binary outcomes, such as cesarean section rate, we calculated the OR and $95 \% \mathrm{Cl}$. For continuous outcomes, such as mean duration to delivery, we calculated the mean difference (MD) with $95 \% \mathrm{Cl}$. The unit of analysis was per woman randomized.

Random effects models were considered appropriate as there were many differences between trials (e.g. participant inclusion criteria, treatment types and dosages) that may potentially influence the size of the treatment effect, although the heterogeneity associated with fixed effects models was always assessed. Fixed effects results were only reported when a random effects analysis could not be performed due to negative "between study" variance for analyses using the inverse variance weighting method.

We assessed the methodological and clinical characteristics of the included studies in to see if they were similar enough for meta-analysis to be clinically meaningful. Heterogeneity was tested by using the $I^{2}$ statistics, where a measurement greater than $50 \%$ was taken to indicate substantial heterogeneity (15). 
We conducted subgroup analyses if sufficient data was available to determine the separate evidence within the subgroups such as primigravida versus multigravida.

For all substantial heterogeneity we might have detected, we explored possible explanations in sensitivity analyses and we have taken all substantial heterogeneity into account in our interpretation of the results.

Sensitivity analyses were done for primary and secondary outcomes to determine whether the conclusions are robust to arbitrary decisions made regarding the eligibility and analysis. Our analyses considered of whether the review conclusions would have differed if eligibility were restricted to studies without high risk of bias, if alternative imputation strategies had been implemented or if the summary effect measure was relative risk rather than odds ratio.

\section{Results}

\section{Literature search}

A flow diagram of the included and excluded studies is shown in Fig 1. The database searches identified 432 records. After removing the duplicates $(n=137)$, two reviewers (MM and SF) screened the titles and abstracts for potentially relevant studies $(n=295)$ independently. Seven full-text RCTs were assessed for eligibility. Three full-text articles excluded due to: one reported non- concurrent administration of oxytocin and Foley catheter, one had a study design not of interest, and the last one was clinical trial registered on clinictrials.gov with no published full text yet. So, four studies were included in the meta-analysis.

\section{Characteristics of studies}

The trails included a total of 430 women in the group of Foley catheter plus oxytocin, and 433 in the Misoprostol group that was published between 1999 and 2016. The characteristics of the studies included in the meta-analysis are shown in table 1. Out of the four included studies, three were conducted in USA, (17-19), and one in Nigeria (20). In general, the included participants had singleton pregnancies with an unfavorable cervix, intact membranes, and with the fetus in cephalic presentation in the third trimester of pregnancy. Three studies recruited primi- and multigravida women (17-19). One study enrolled only multigravida women (20). Only in two studies, the main outcomes were reported separately by primi and multigravidas $(17,19)$.

\section{Risk of bias in included studies}

The random sequence and allocation concealment were reasonable in most trials $(100 \%$ and $60 \%$, respectively). The lack of blinding in most of the study trials was likely to be the main source of bias; only one trial reported an adequate description of blinding of the study subjects (13). Selective reporting was reasonable (60\%) but incomplete outcome data was considered a high risk of bias in most of the included trials (Figure 2).

\section{Effects of interventions}




\section{Cesarean section rate}

\section{A. Foley plus oxytocin vs. vaginal Misoprostol}

As indicated in Fig 3, there were 430 participants in Foley plus oxytocin groups and 433 in misoprostol groups. The overall cesarean section rate in Foley plus oxytocin group was not significantly different from Misoprostol groups (OR: 091, 95\% Cl: 0.71-1.18). Subgroup analysis of primiparous and multiparous women didn't show any differences in the rate of cesarean section in both groups $(\mathrm{OR}=1.00$; $95 \% \mathrm{Cl}: 0.63,1.60$, in primiparous vs. $\mathrm{OR}=0.68 ; 95 \% \mathrm{Cl}: 0.33,1.42$ in multiparous).

\section{B. Foley+ oxytocin vs. Foley + vaginal Misoprostol}

Only one study compared the cesarean rate between Foley plus oxytocin versus Foley + vaginal Misoprostol (17). The result regarding the cesarean rate was not different between the two groups (OR = $1.14 ; 95 \% \mathrm{Cl}: 0.66,1.98)$. However, in the subgroup analysis of multiparous women vs. primiparous, there was an increased rate of cesarean section in Foley plus oxytocin group in multiparous women.

\section{Foley + oxytocin versus oral Misoprostol}

In one study cesarean section rate was assessed between Foley plus oxytocin group versus oral Misoprostol. The overall cesarean section rate was not significantly different between the two groups (OR $=0.99 ; 95 \% \mathrm{Cl}: 0.51,1.91)(19)$.

\section{Mean duration of induction to delivery}

Three studies ( $n=554$ women) were included in the meta- analysis of mean duration of induction to delivery in two groups (Fig 4). Foley plus oxytocin decreased the mean duration of induction to delivery compared to vaginal Misoprostol (MD $=-6.74 ; 95 \% \mathrm{Cl}-9.20,-4.28)$ in nuligravidae but not in multigravidae $(\mathrm{MD}=-0.87 ; 95 \% \mathrm{Cl}-5.09,3.35)$. One study compared mean duration from insertion of catheter to delivery between Foley plus oxytocin versus Foley plus vaginal Misoprostol. The overall mean duration in Foley plus oxytocin group was not significantly different from Foley plus vaginal Misoprostol group $(M D=1.08$; 95 \% Cl: $-0.78,2.95)(17)$.

\section{Delivery in less than 12 hours from induction}

As indicated in Fig 5, there were 67 participants in Foley plus oxytocin group and 39 participants in vaginal Misoprostol group. Delivery in less than 12 hours from induction in Foley plus oxytocin group was significantly more than that in the Misoprostol groups ( $\mathrm{OR}=2.08 ; 95 \% \mathrm{Cl}: 1.43,3.02)$. Subgroup analysis of primiparous women showed significant decrease in the time of delivery in Foley + oxytocin group $(\mathrm{OR}=2.21 ; 95 \% \mathrm{Cl}: 1.18,4.15)(17,18)$.

Comparison between Foley plus oxytocin versus Foley plus vaginal Misoprostol showed the overall mean duration in Foley plus oxytocin group was not significantly different from Foley plus vaginal Misoprostol group (OR $=0.85 ; 95 \% \mathrm{Cl}:[0.51,1.41], \mathrm{P}=0.53)$. (17). 


\section{Adverse events}

Two studies with 418 participants reported admission in neonatal intensive care unit (NICU) rate $(17,18)$. There was no significant difference between Foley plus oxytocin and vaginal Misoprostol groups (OR = $0.72 ; 95$ \% Cl: 0.0.41, 1.24) (Fig 6).

\section{Neonatal sepsis}

Two studies (involving 428 women) were included in the meta- analysis of neonatal sepsis $(17,18)$. There was no significant difference between Foley plus oxytocin and vaginal Misoprostol groups $(\mathrm{OR}=0.64 ; 95$ $\%$ Cl: $0.24,1.75)$.

\section{Hyper-stimulation or terbutaline use}

Two studies (involving 418 women) were included in the meta-analysis for assessment hyper-stimulation or terbutaline use $(17,18)$. There was no significant difference on the rate of hyper-stimulation or terbutaline use between Foley plus oxytocin and vaginal Misoprostol groups (OR $=0.069 ; 95 \% \mathrm{Cl}$ : 0.38, 1.25).

\section{Others adverse outcomes}

There was only one study that assessed the rate of chorioamnionitis and endometritis between Foley plus oxytocin and vaginal Misoprostol groups (17). The result of analysis showed significantly higher rate of chorioamnionitis in vaginal Misoprostol group compared to Foley plus oxytocin (OR $=2.35 ; 95 \%$ $\mathrm{Cl}: 1.02,5.39)$. However, there was no significant difference between two groups regarding the rate of endometritis (OR $=1.37 ; 95 \% \mathrm{Cl}: 0.67,2.82)$.

\section{Discussion}

This systematic review was conducted to assess the effect of Foley catheter plus oxytocin vs. misoprostol for induction of labor. Our results indicated that the rate of cesarean section was not different between two groups of administration Foley catheter plus oxytocin with misoprostol.

Insertion of Foley catheter is a mechanical method for labor induction in which, cervix is dilated via increase in uterine receptors and progressive response to oxytocin and prostaglandin (21). Some studies showed that with recruiting women with Bishop Score 3 to 6 , there is no significant difference between labor induction and expectant management of labor regarding cesarean section rate and adverse events in mothers and babies (15). The Bishop Score in time of labor induction is an important parameter. Misoprostol is recommended for labor induction by the World Health Organization (22), International Federation of Gynecology and Obstetrics (23), and the Society of Obstetricians and Gynecologists of Canada (24). Studies showed that using oral Misoprostol is preferable to vaginal suppository for labor induction (25). In the current systematic review, all included studies recruited women with term pregnancy and Bishop Score 3-6 and used oral or vaginal Misoprostol for labor induction. 
Our results regarding mean duration from induction to delivery revealed that Foley + oxytocin could significantly decrease the mean duration from induction to delivery. This effect was more obvious in primigravida vs. multigravida women. Studies in which they used misoprostol vs. Foley catheter revealed that Misoprostol in labor induction resulted in better outcomes in terms of normal vaginal delivery rate (26). In line with our findings, the mechanical methods of induction were found to be safe even in cases with intrauterine growth restriction (27). Interestingly, the mechanical methods were associated with lower rates of tachysystoles and the rates of cesarean sections were comparable to the pharmacological methods (27). Similar to what has been found in our review, the mechanical methods were found to be associated with a short period of induction of labor and no difference was found between concurrent Foley catheter and oxytocin vs. Misoprostol and Foley catheter (28).

Results of this systematic review showed that concurrent use of Foley + oxytocin could significantly increase delivery in less than 12 hours from induction compared to Misoprostol. Other studies also revealed that using Foley catheter is safe as spontaneous labor. The Foley mechanism for ripening cervix is defined as direct mechanical pressure on cervix and cervical dilation as a result and also the local secretion of prostaglandins (29). Studies showed that the level of prostaglandin in women who used Foley catheter for cervical ripening, increased and this increase has continued with the level of higher cervical dilation (30).

\section{Strengths and limitations of the study}

Although there are many studies that evaluated the effect of oxytocin vs. Misoprostol or vs. Foley catheter for labor induction, the information regarding comparison of Foley catheter plus oxytocin vs. Misoprostol for induction of labor is scarce. This is a first systematic review that evaluated the impact of concurrent use of Foley catheter and oxytocin vs. Misoprostol in primi and multipara women. The other strengths of this study are; we used the Cochrane methodology for assessing the risk of bias, use of systematic method for search of studies and use of RevMan for analyzing data. In this study, because the number of included studies was small (four studies), no definite conclusion can be made about the use of Foley catheter plus oxytocin.

\section{Conclusions}

This systematic review showed that although the rate of cesarean section and vaginal delivery was not different between two groups of concurrent use of Foley catheter plus oxytocin and vaginal Misoprostol, the mean time from induction to delivery and delivery less than 12 hours from induction in Foley plus oxytocin was significantly better than that in the Misoprostol alone. Concurrent use of Foley plus oxytocin in term pregnancies which need labor induction has a potential benefit as it showed faster delivery without increasing side effects.

\section{Declarations}


Acknowledgement

$\mathrm{N} / \mathrm{A}$

Funding

No funding was received for this research

\section{Availability of data and materials}

Data sharing is not applicable for this study. There is no datasets were used for this study.

\section{Authors' contributions}

Conceptualization: MAA, PA, SF, SJ

Search and screening: MAA

Analyses and interpretation: MAA, PA and SJ

Writing and finalizing the manuscript: FS, PA and SJ.

All authors read and approved the final version of the study.

Ethics approval and consent to participate

N/A

Consent for publication

$\mathrm{N} / \mathrm{A}$

Competing interests: Parvin Abedi and Shayesteh Jahanfar are both associate editors of BMC Pregnancy and Childbirth and other than this; the authors declare that they have no competing interests.

\section{Abbreviations}

Cl: Confidence Interval

MD: Mean Difference

RCT: Randomised Controlled Trial

PRISMA: Preferred Reporting Items for Systematic Reviews and Meta-Analyses

NICU: neonatal intensive care unit 


\section{References}

1. Mishanina E, Rogozinska E, Thatthi T, Uddin-Khan R, Khan KS, Meads C. Use of labour induction and risk of cesarean delivery: a systematic review and meta-analysis. CMAJ. 2014;186(9):665-73.

2. Berns SD, Kott A. Toward Improving the Outcome of Pregnancy III: Enhancing Perinatal Health Through Quality, Safety and Performance Initiatives. White Plains, NY: March of Dimes National Foundation. 2010.

3. Bernardes TP, Broekhuijsen K, Koopmans CM, Boers KE, van Wyk L, Tajik P, et al. Caesarean section rates and adverse neonatal outcomes after induction of labour versus expectant management in women with an unripe cervix: a secondary analysis of the HYPITAT and DIGITAT trials. BJOG. 2016;123(9):1501-8.

4. Lim CE, Ng RW, Xu K. Non-hormonal methods for induction of labour. Curr. Opin. Obstet. Gynecol. 2013;25(6):441-7.

5. Gu N, Ru T, Wang Z, Dai Y, Zheng M, Xu B, et al. Foley Catheter for Induction of Labor at Term: An Open-Label, Randomized Controlled Trial. PloS one. 2015;10(8):e0136856.

6. Kavanagh J, Kelly AJ, Thomas J. Sexual intercourse for cervical ripening and induction of labour. The Cochrane database of Systematic Reviews. 2001;2001(2):Cd003093.

7. Nishi D, Shirakawa MN, Ota E, Hanada N, Mori R. Hypnosis for induction of labour. The Cochrane database of Systematic Reviews. 2014(8):Cd010852.

8. Kavanagh J, Kelly AJ, Thomas J. Breast stimulation for cervical ripening and induction of labour. The Cochrane database of Systematic Reviews. 2001(4):Cd003392.

9. Gilstrop M, Sciscione A. Induction of labor-pharmacology methods. SEMIN PERINATOL. 2015;39(6):463-5.

10. Yazdizadeh H, Abedi P, Najar S, Angali KA. The impact of isosorbide mononitrate on cervical ripening and labor induction in primiparous women with term pregnancy: A double-blind, randomized, controlled trial. Iranian J Nursing Midwifery Res. 2013;18(3):246-50.

11. Fox NS, Saltzman DH, Roman AS, Klauser CK, Moshier E, Rebarber A. Intravaginal Misoprostol versus Foley catheter for labour induction: a meta-analysis. BJOG. 2011;118(6):647-54.

12. Lanka S, Surapaneni T, Nirmalan PK. Concurrent use of Foley catheter and Misoprostol for induction of labor: a randomized clinical trial of efficacy and safety. J Obstet Gynaecol rRes. 2014;40(6):152733.

13. ACOG Practice Bulletin No. 107: Induction of labor. Obstet Gynecol. 2009;114(2 Pt 1):386-97.

14. Conway A, Clarke M, Treweek S, Schünemann H, Santesso N, Morgan R, et al. Summary of findings tables for communicating key findings of systematic reviews: Protocols. Cohrane Database of Systematic Reviews. 2017.

15. Higgins JPT, Green S, eds. The Cochrane Collaboration. Cochrane Handbook for Systematic Reviews of Interventions. Version 5.1.0. https://handbook-5-1. cochrane.org. Updated March 2011. Accessed April 3, 2017. 
16. Higgins JPT, Altman DG. Assessing Risk of Bias in Included Studies. Cochrane Handbook for Systematic Reviews of Interventions. 2008:187-241.

17. Levine LD, Downes KL, Elovitz MA, Parry S, Sammel MD, Srinivas SK. Mechanical and Pharmacologic Methods of Labor Induction: A Randomized Controlled Trial. Obstet Gynecol. 2016;128(6):1357-64.

18. Culver J, Strauss RA, Brody S, Dorman K, Timlin S, McMahon MJ. A randomized trial comparing vaginal Misoprostol versus Foley catheter with concurrent oxytocin for labor induction in nulliparous women. Am. J. Perinatol. 2004;21(3):139-46.

19. Abramovici D, Goldwasser S, Mabie BC, Mercer BM, Goldwasser R, Sibai BM. A randomized comparison of oral Misoprostol versus Foley catheter and oxytocin for induction of labor at term. AM J OBSTET GYNECOL. 1999;181(5 Pt 1):1108-12.

20. Garba I, Muhammed AS, Muhammad Z, Galadanci HS, Ayyuba R, Abubakar IS. Induction to delivery interval using transcervical Foley catheter plus oxytocin and vaginal Misoprostol: A comparative study at Aminu Kano Teaching Hospital, Kano, Nigeria. Ann. Afr. Med. 2016;15(3):114-9.

21. Diederen M, Gommers J, Wilkinson C, Turnbull D, Mol B. Safety of the balloon catheter for cervical ripening in outpatient care: complications during the period from insertion to expulsion of a balloon catheter in the process of labour induction: a systematic review. BJOG. 2018;125(9):1086-95.

22. WHO. WHO Recommendations for Induction of Labour. Geneva: World Health Organization. 2011.

23. FIGO.[http://www.figo.org/sites/default/files/uploads/projectpublications/Miso/Misoprostol_Recommended\%20Dosages\%202012.pdf]Accessed 7March 2019.

24. Leduc D, Biringer A, Lee L, Dy J. Clinical Practice Obstetrics Committee, Society of Obsetricians and Gynaecologists of Canada. Induction of labour. SOGC Clinical Practice Guideline No. 296, September 2013. J Obstet Gynaecol Can. 2013;36:248-52.

25. Alfirevic Z, Weeks A. Oral Misoprostol for induction of labour. Cochrane Database of Systematic Reviews. 2014; https://doi.org/10.1002/14651858.CD001338

26. Vahid Roudsari F, Ayati S, Ghasemi M, Hasanzadeh Mofrad M, Shakeri MT, Farshidi F, et al. Comparison of vaginal Misoprostol with foley catheter for cervical ripening and induction of labor. IJPR. 2011;10(1):149-54.

27. Familiari A, Khalil A, Rizzo G, Odibo A, Vergani P, Buca D, et al. Adverse intrapartum outcome in pregnancies complicated by small for gestational age and late fetal growth restriction undergoing induction of labor with Dinoprostone, Misoprostol or mechanical methods: A systematic review and meta-analysis. Eur J Obstet Gynecol Reprod Biol. 2020;252:455-67.

28. Orr L, Reisinger-Kindle K, Roy A, Levine L, Connolly K, Visintainer P, et al. Combination of Foley and prostaglandins versus Foley and oxytocin for cervical ripening: a network meta-analysis. AM J OBSTET GYNECOL. 2020.

29. Greenberg V, Khalifeh A. Intracervical Foley balloon catheter for cervical ripening and labor induction: A review. SEMIN PERINATOL. 2015;39(6):441-3.

30. Manabe $Y$, Manabe A, Takahashi A. F prostaglandin levels in amniotic fluid during balloon-induced cervical softening and labor at term. Prostaglandins. 1982;23(2):247-56. 


\section{Table}

Table 1: Characteristics of included studies 


\section{Reference Culver, 2004}

Methods Parallel design -Two arms -Phase III -Country: USA, North Carolina Women's Hospital (Chapel Hill, NC) and WakeMed Hospital (Raleigh, NC). -Unit of randomization: participant -Analysis unit: participant

Participants -Enrolled: 173 pts Misoprostol alone: 84

-Randomized: 1) Foley + oxytocin: 89 pts

- All patients were nulliparous. Inclusion criteria: Nulliparity, gestational age >28 weeks, a Bishop score $<6$, and intact membranes.

Exclusion criteria: (1) a previous delivery past 20 weeks, (2) multiple gestation, (3) non-cephalic presentation, (4) previous uterine surgery, (5) non-reassuring fetal heart rate (FHR) tracing, (6) more than eight spontaneous contractions in the hour preceding randomization, (7) latex allergy, or (8) any contraindication to vaginal delivery.

Interventions 1. Foley catheter + Oxytocin: $18 \mathrm{~F}$ Foley catheter with $30-\mathrm{mL}$ balloon was placed through the cervical canal. The balloon was then inflated in the lower uterine segment with $30 \mathrm{~mL}$ of sterile water and the Foley catheter taped under gentle traction to the patient's inner thigh. Intravenous oxytocin was then started at $2 \mathrm{mU} / \mathrm{min}$ and increased 1 to $2 \mathrm{mU} / \mathrm{min}$ every 15 to 30 minutes to a maximum of $20 \mathrm{mU} / \mathrm{min}$. Maximum oxytocin doses could exceed $20 \mathrm{mU} / \mathrm{min}$ by physician order.

2. Intravaginal Misoprostol: misoprostol $25 \mathrm{mg}$ intra-vaginally every 4 hours up to a maximum of six doses. Tablets $(100 \mathrm{mg}$ ) were cut into fourths by the nurse and one fourth tablet was placed into the posterior fornix by the physician. Subsequent doses were withheld if the patient was having three or more contractions in 10 minutes or non-reassuring FHR tracing. Oxytocin augmentation was withheld until 4 hours after placement of the last misoprostol dose. If oxytocin was used, the protocol listed above was followed.

Outcomes - Primary: CS rate

-Secondary: indications for cesarean delivery, time from induction to delivery, abnormalities of labor such as tachysystole, hyper stimulation, abnormal FHR tracings; intrapartum and postpartum fevers and use of antibiotics; estimated blood loss; and blood transfusions, Apgar scores, neonatal resuscitation requirements, admission to the neonatal intensive care unit, meconium aspiration, hyperbilirubinemia, sepsis (culture proven or clinically suspected), death.

\section{Reference Levine, 2016}

Methods -Parallel design $\quad$-Four arm -Phase III

-Country: USA, University of Pennsylvania. the Hospital of the University of

Pennsylvania

-Unit of randomization: participant

-Analysis unit: participant

Participants Enrolled: 491 pts

-Randomization: 1) Foley alone: 123 pts, 2) Misoprostol alone: 120 pts, 3) Foley + misoprostol: 123 pts,

4) Foley + oxytocin: $125 \mathrm{pts}$

-Nulliparous: N (\%):1) misoprostol alone :70 (58.3), 2) Misoprostol + Foley: 73

$(59.4,3)$ 3) Foley alone: 73 (59.4), 4) Foley + oxytocin: 74 (59.2) 
Inclusion Criteria: at least 18 years of age with a full term ( $\geq 37$ weeks), singleton gestation in cephalic presentation. Both nulliparous and multiparous women were included. Women were required to have intact membranes, a Bishop score of $\leq 6$ and cervical dilation $\leq 2 \mathrm{~cm}$ to be eligible

Exclusion Criteria: there was a contraindication to a vaginal delivery or to misoprostol, fetal demise, or major fetal anomaly. Non-English speaking women, women with HIV, and women with medical conditions requiring an

Interventions 1. Foley + oxytocin: $18 \mathrm{~F}$ Foley catheter with $30 \mathrm{cc}$ balloon inflated with $60 \mathrm{cc}$ sterile water and deflated and removed after $12 \mathrm{hrs}$. if not spontiously dropped. Oxytocin was initiated concurrently at the start of induction. Oxytocin protocol: 2 mill units/minute of oxytocin increasing by 2 mill units every 15 minutes until regular uterine contractions occur. Forty mill units of oxytocin is considered the maximum dose with no limit as to the length of time a participant can remain at 40 milliunits.

2. Intravaginal Misoprostolalone: 25 micrograms of misoprostol per vagina every 3 hours, repeated up to five additional times for a maximum of 24 hours, Oxytocin was initiated if there was a contraindication to another misoprostol dose or if additional cervical ripening was not indicated.

3) Foley catheter as in intervention (Foley alone group) and oxytocin was initiated when the catheter either dropped spontiously or removed after $12 \mathrm{hrs}$. by the care giver

4) Foley + intravaginal misoprostol as described above for each one of the interventions.

Outcomes

-Primary: time to delivery (hours)

-Secondary: Cesarean delivery rate, time to delivery censored for cesarean section, time to active labor (defined as dilatation $\geq 5 \mathrm{~cm}$ ), delivery within 12 hours, delivery within 24 hours, and maternal length of stay (defined as length of time from admission for induction to discharge postpartum, days) and indication for cesarean delivery, 3rd/4th degree perineal laceration, blood transfusion, endometritis, wound separation-infection (defined by the need for additional wound closure or the need for antibiotics), venous thromboembolism, hysterectomy, intensive care unit admission, or death. Other maternal secondary outcomes analyzed were chorioamnionitis (defined by the presence of maternal fever $\geq 100 \cdot 4^{\circ} \mathrm{F}$ in the presence of maternal or fetal tachycardia or fundal tenderness), use of terbutaline, intrauterine pressure catheter, amnioinfusion, or analgesia use. neonatal morbidity outcome was pre-specified to include $\geq 1$ of the following prior to neonatal discharge: severe respiratory distress syndrome (defined as intubation and mechanical ventilation for a minimum of 12 hours), culture proven-presumed neonatal sepsis, neonatal blood transfusion, hypoxic ischemic encephalopathy, intraventricular hemorrhage grade 3 or 4, necrotizing enterocolitis, or receipt of head cooling. Other neonatal outcomes analyzed were neonatal intensive care unit (NICU) admission, NICU admission $>48$ hours, and neonatal length of stay (days).

\section{Reference Garba, 2016}

Methods -Parallel design $\quad$-Two arm -Phase III

-Country: Nigeria: Aminu Kano Teaching Hospital, From February 01 to May 30, 2015

-Unit of randomization: participant

-Analysis unit: participant

Participants -Number enrolled: 136 pts

$\begin{array}{lll}\text {-Randomized: } & \text { 1) Foley + oxytocine:66 pts. } & \text { 2) misoprostol alone: } 70\end{array}$ 
-Primigravida: N (\%): All patients were Multigravida

Inclusion criteria:postdate singleton pregnant multigravida

Exclusion criteria:not mentioned

Interventions 1. Foley + oxytocin: the details did not mentioned

2. Intravaginal misoprostol alone: the details did not mentioned

Outcomes -Primary outcomes:Mean induction to delivery time

-Secondary outcomes:CS rate, Failed induction rate, Failure to progress rate, The APGAR scores, maternal vital signs, estimated blood loss

\section{Reference Abramovici, 1999}

Methods $\quad-R C T \quad$ - two arms $\quad$-Phase III

Country: USA, Division of Maternal-Fetal Medicine, Department of Obstetrics and Gynecology, University of Tennessee.

-Unit of randomization: participant $\quad$-Analysis unit: participant

\section{Participants -Enrolled: 197 pts}

$\begin{array}{lll}\text {-Randomized: } & \text { 1) Foley +oxytocin: } 99 \text { pts, } & \text { 2) Oral misoprostol alone: } 98\end{array}$ pts

-Primigravida: N (\%): $\quad$ 1) Oxytocin + Foley: 57 pts, $\quad$ 2) misoprostol: 58 pts

Inclusion criteria:Women at term (37 weeks' gestation) with a singleton live fetus in cephalic presentation, had intact membranes, had a Bishop score of 5 or less, and were not in labor (<3 uterine contractions in 10 minutes).

Exclusion criteria: any of the following:

a no reassuring fetal heart rate tracing, multifetal gestation, previous cesarean delivery or previous uterine surgery, ruptured membranes, placenta Previa, active genital herpes, maternal asthma, sickle cell disease, or known hypersensitivity to prostaglandins.

Interventions 1. Foley + oxytocin:

Foley + oxytocin: In patients without any cervical dilation, a Foley catheter was inserted into the cervix under direct visualization. A speculum was placed into the vagina, and the cervix was cleaned with an antiseptic solution. A 16F balloon-tipped Foley catheter (C.R. Bard, Inc., Covington, Ga) was passed through the cervix beyond the internal os and was inflated with $30 \mathrm{~mL}$ of sterile sodium chloride solution. The catheter was taped under gentle traction to the inner aspect of the patient's thigh. The balloon was deflated, and the device was removed after 12 hours if it had not been spontaneously expelled,

Oxytocin: Intravenous oxytocin was started at $1 \mathrm{mU} / \mathrm{min}$ and increased by $2 \mathrm{mU} / \mathrm{min}$ at 30-minute intervals until adequate uterine activity was maintained (3 contractions in 10 minutes). The maximum dose of oxytocin allowed was $36 \mathrm{mU} / \mathrm{min}$.

2. oral Misoprostol alone: 50 - microgram tablet orally every 4 hours over a 24-hour period up to a maximum dose of $300 \mathrm{mg}$. 
Outcomes 1. Primary: delivery within 24 hours from the start of induction

2. Secondary: induction-to-delivery time, time to active labor, incidence of cesarean delivery, indications for cesarean delivery, uterine hyper stimulation, and neonatal outcome eg meconium, NICU admiration, Apgar score at $1 \mathrm{~min}$ and $5 \mathrm{~min}$, Maternal SEs eg, chorioamnionitis

\section{Figures}




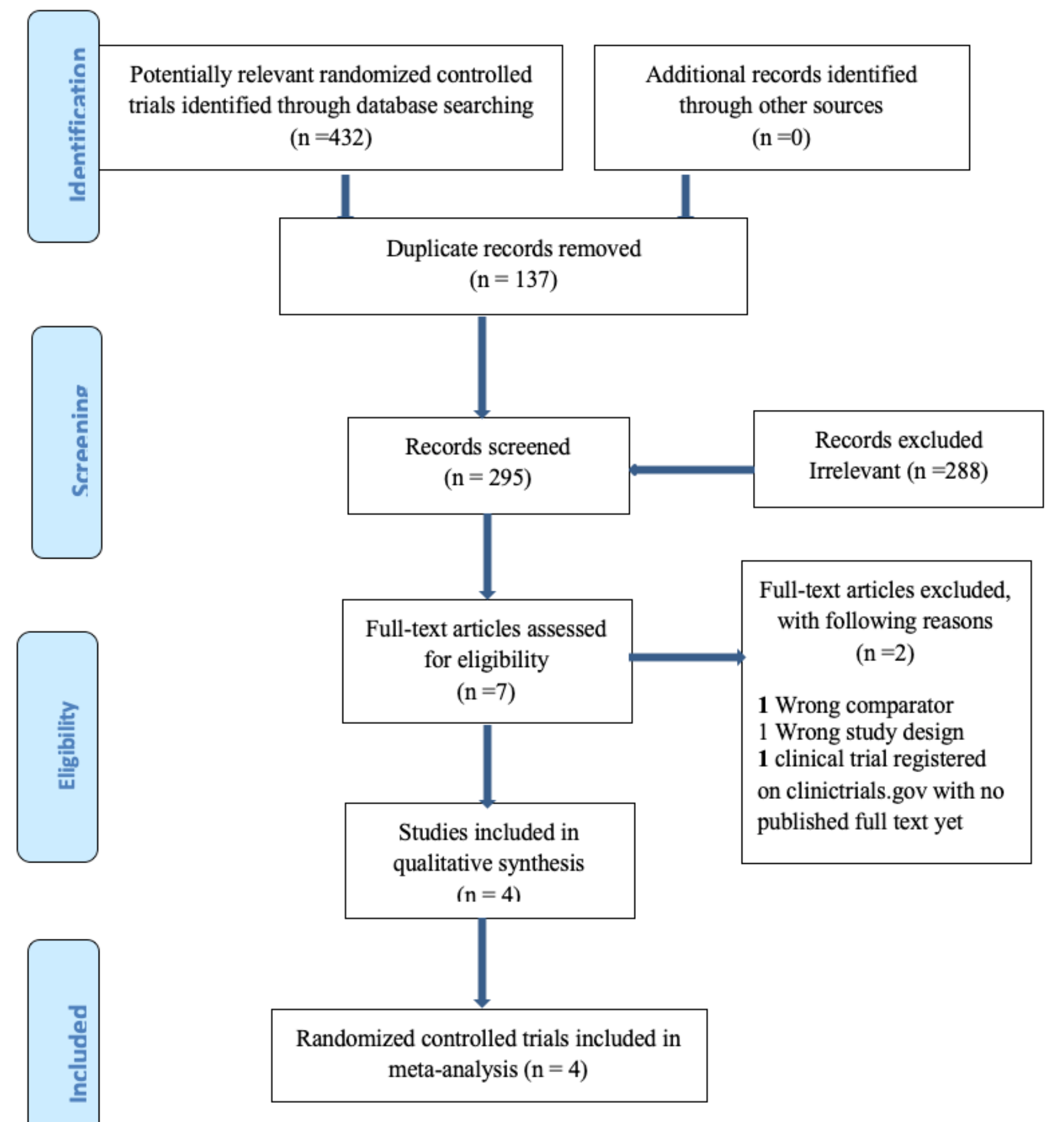

Figure 1

PRISMA Chart 


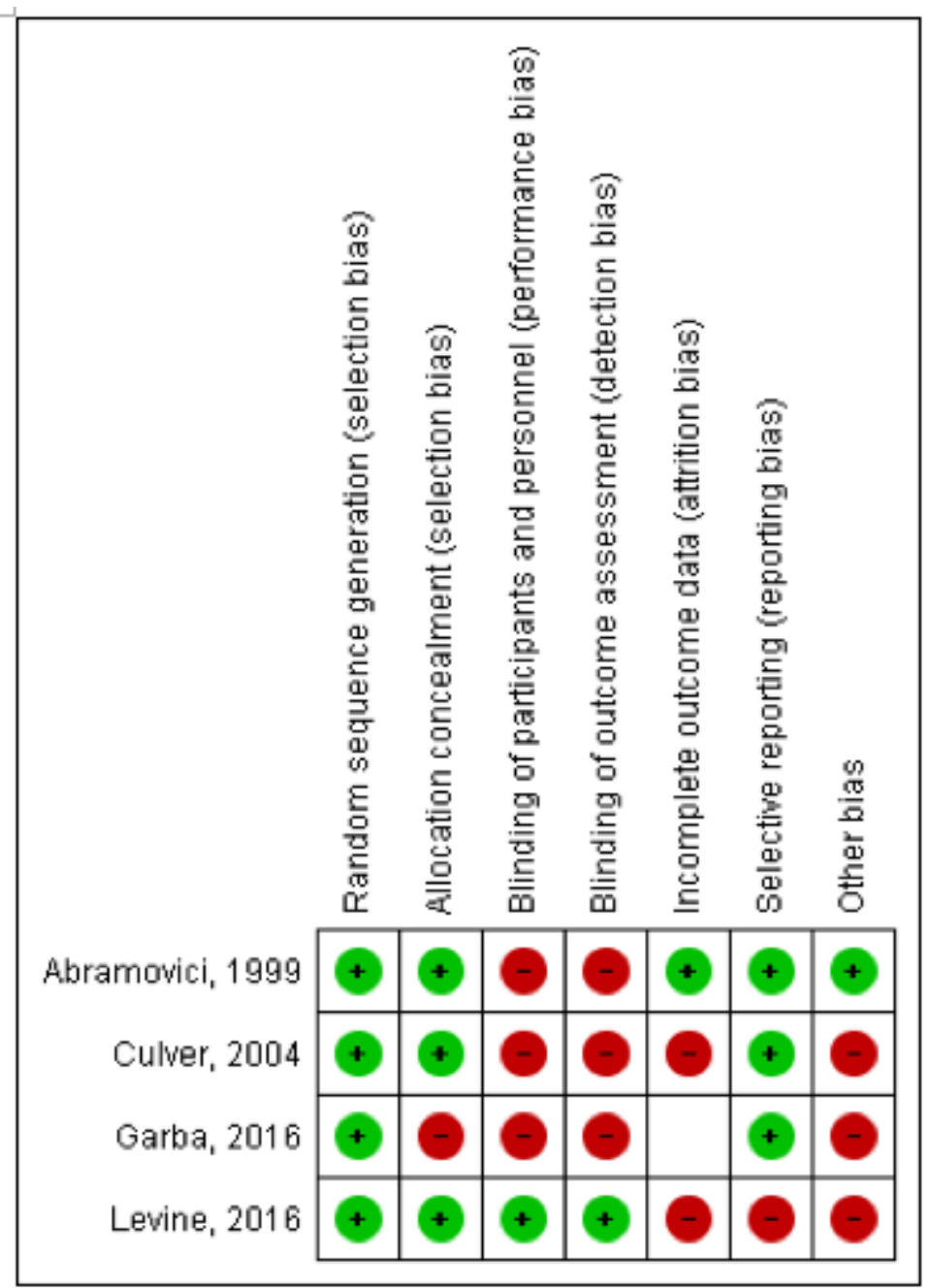

Figure 2

Risk of bias summary: review authors' judgments about each risk of bias item for each included study 
Study or Subgroup

foley catheter+ oxytocin Misoprostol

Odds Ratio

Odds Ratio

7.1.1 All Women

$\begin{array}{lrrrrrr}\text { Abramovici, 1999 } & 23 & 99 & 23 & 98 & 14.3 \% & 0.99[0.51,1.91] \\ \text { Culver, 2004 } & 24 & 89 & 28 & 84 & 16.9 \% & 0.74[0.38,1.42] \\ \text { Garba, 2016 } & 6 & 66 & 14 & 70 & 9.9 \% & 0.40[0.14,1.11] \\ \text { Levine, 2016 } & 38 & 125 & 29 & 120 & 16.6 \% & 1.37[0.78,2.41] \\ \text { Subtotal (95\% Cl) } & & 379 & & 372 & 57.7 \% & 0.92[0.66,1.29]\end{array}$

Total events 91

Heterogeneity. $\mathrm{Chi}^{2}=4.93, \mathrm{df}=3(\mathrm{P}=0.18) ; \mathrm{I}^{2}=39 \%$

Test for overall effect: $Z=0.47(P=0.64)$

7.1.2 Nuligravidum

$\begin{array}{lcrrrrr}\text { Culver, } 2004 & 24 & 89 & 28 & 84 & 16.9 \% & 0.74[0.38,1.42] \\ \text { Levine, } 2016 & 30 & 74 & 23 & 70 & 11.3 \% & 1.39[0.70,2.75] \\ \text { Subtotal }(95 \% \mathrm{CI}) & & \mathbf{1 6 3} & & \mathbf{1 5 4} & \mathbf{2 8 . 2 \%} & \mathbf{1 . 0 0 [ 0 . 6 3 , 1 . 6 0 ]} \\ \text { Total events } & 54 & & 51 & & & \end{array}$

Heterogeneity. $\mathrm{Chi}^{2}=1.74, \mathrm{df}=1(\mathrm{P}=0.19) ; \mathrm{I}^{2}=43 \%$

Test for overall effect: $Z=0.00(P=1.00)$

\subsubsection{Multigravidum}

$\begin{array}{lrrrrrr}\text { Garba, } 2016 & 6 & 66 & 14 & 70 & 9.9 \% & 0.40[0.14,1.11] \\ \text { Levine, 2016 } & 8 & 51 & 6 & 50 & 4.1 \% & 1.36[0.44,4.26] \\ \text { Subtotal (95\% Cl) } & & 117 & & \mathbf{1 2 0} & \mathbf{1 4 . 0 \%} & 0.68[0.33,1.42] \\ \text { Total events } & 14 & & 20 & & & \end{array}$

Heterogeneity $\mathrm{Chi}^{2}=2.47, \mathrm{df}=1(\mathrm{P}=0.12) ; \mathrm{I}^{2}=59 \%$

Test for overall effect: $Z=1.02(P=0.31)$

Total $(95 \% \mathrm{Cl})$

659

Total events

159

$646100.0 \%$

$0.91[0.71,1.18]$

Heterogeneity $\mathrm{Chi}^{2}=9.80, \mathrm{df}=7(\mathrm{P}=0.20) ; \mathrm{I}^{2}=29 \%$ 165

Test for overall effect: $Z=0.72(P=0.47)$

Test for subaroup differences: $\mathrm{Ch}^{\mathrm{z}}=0.76, \mathrm{df}=2(\mathrm{P}=0.68), \mathrm{I}^{\mathrm{z}}=0 \%$

M-H, $\mathrm{Fixed,95 \% Cl}$

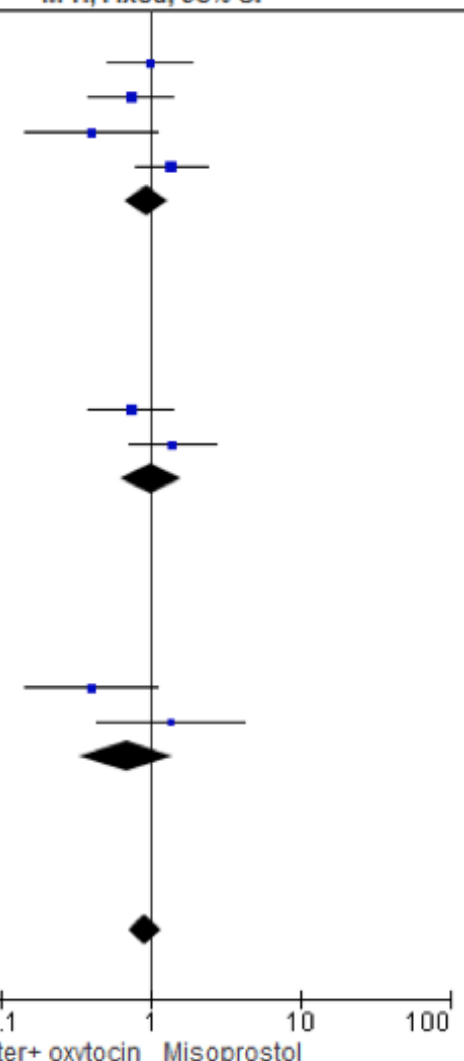

\section{Figure 3}

Forest plot of the cesarean section rate in Foley catheter plus oxytocin vs. Misoprostol groups using fixed effect

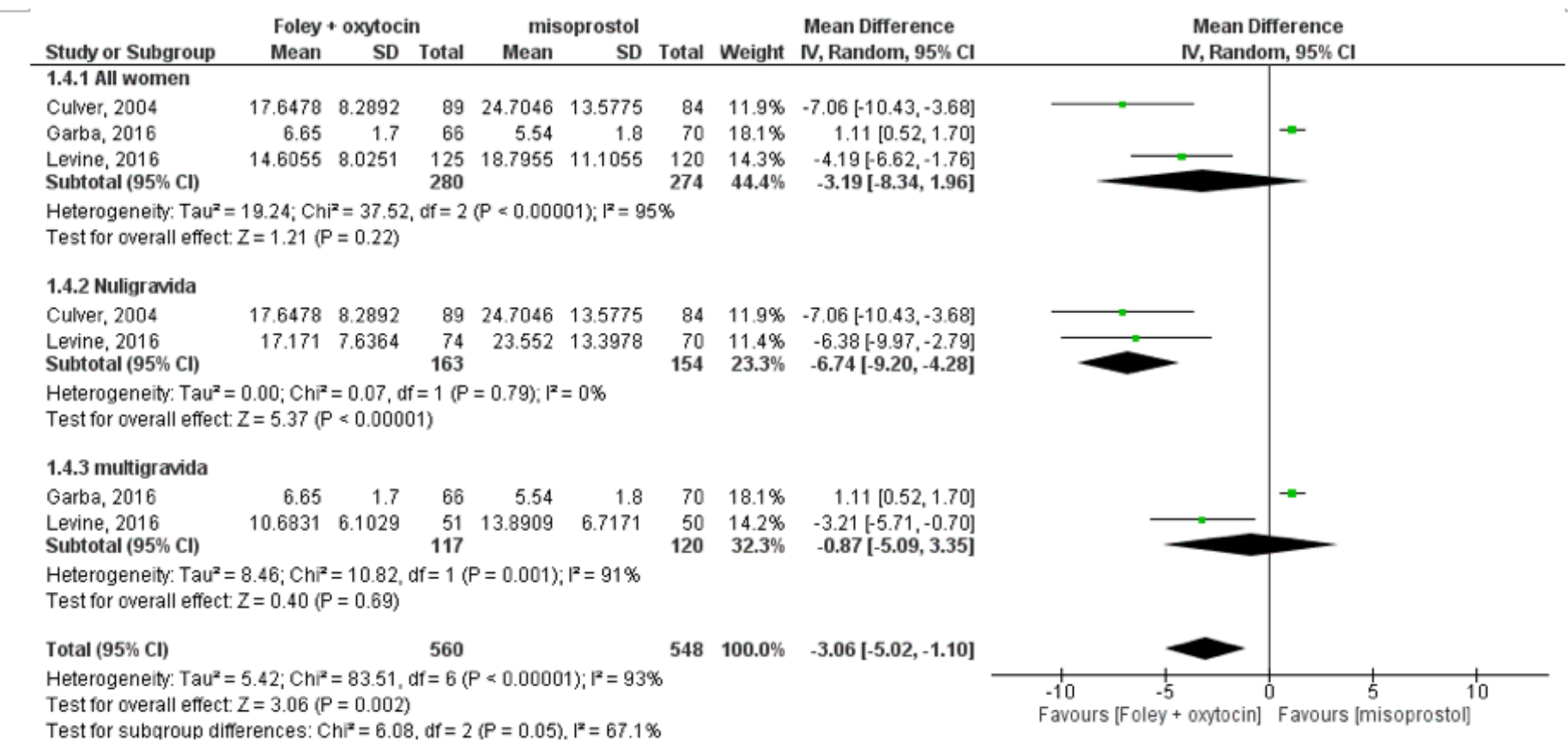

\section{Figure 4}


Forest plot of mean duration to delivery in Foley catheter plus oxytocin and Misoprostol groups using random effect

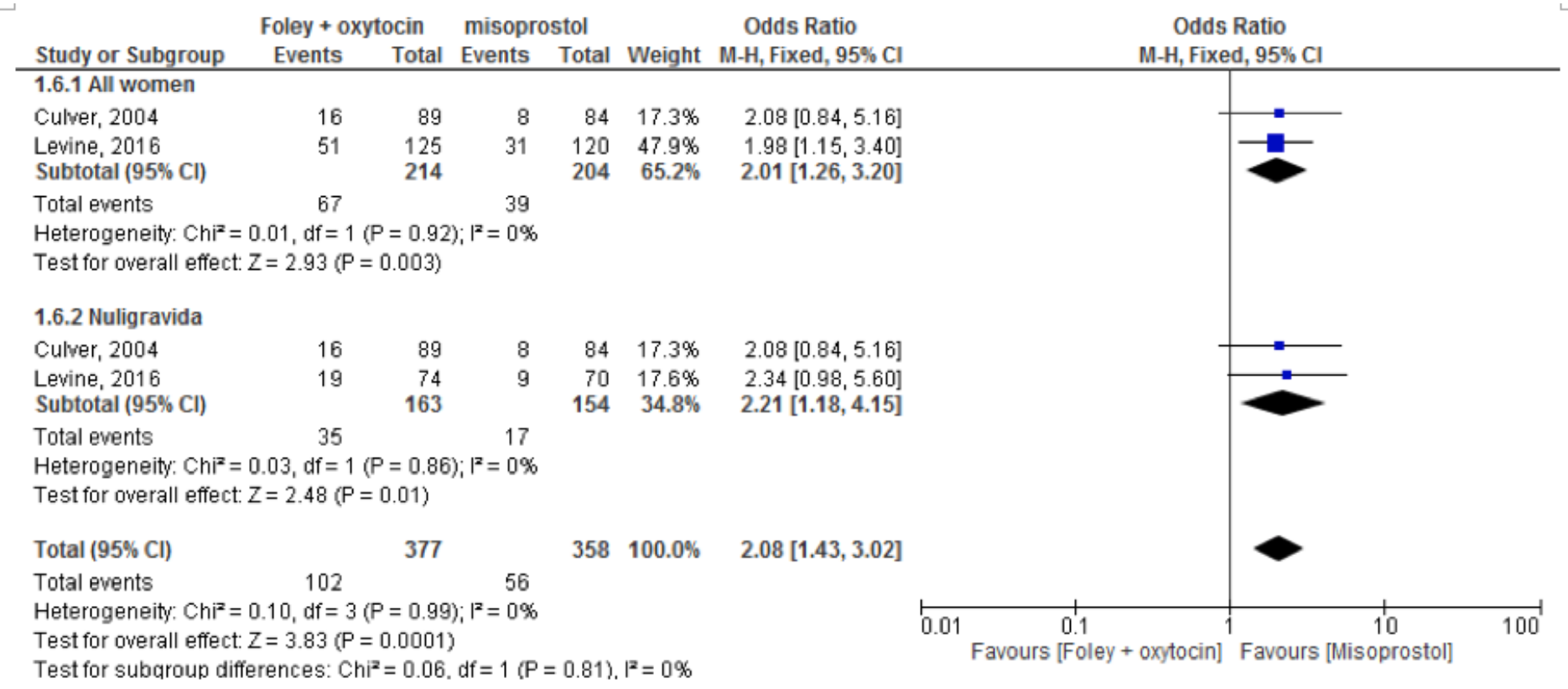

Figure 5

Forest plot of delivery less than 12 hours in Foley catheter plus oxytocin vs. Misoprostol using fixed effect

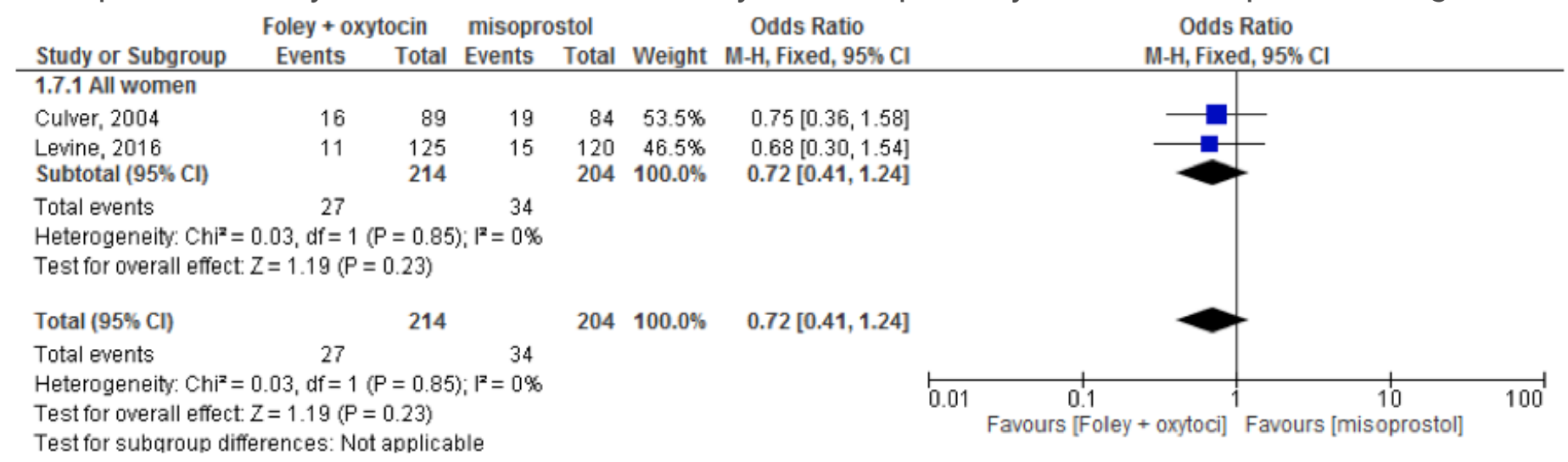

\section{Figure 6}

Forest plot of admission to NICU in Foley catheter plus oxytocin and Misoprostol using fixed effect

\section{Supplementary Files}

This is a list of supplementary files associated with this preprint. Click to download.

- Supplementalmaterial1Searchstrategies.docx 